\title{
Surface and Electric Field Imaging by Newly Designed Atomic-Resolution STEM
}

\author{
Ryo Ishikawa $^{1}$, Naoya Shibata ${ }^{1,2}$ and Yuichi Ikuhara ${ }^{1,2}$ \\ 1. Institute of Engineering Innovation, University of Tokyo, Tokyo, Japan. \\ 2. Nanostructured Research Laboratory, Japan Fine Ceramics Center, Nagoya, Japan.
}

Aberration correction in electron optics has significantly improved the spatial resolution and sub-angstrom resolution is routinely available. Such electron microscopes have become an essential tool to identify the materials structures. However, the demonstrated spatial resolution is limited in two dimensions (or projection) and it is still challenging to investigate $3 \mathrm{D}$ atomic structures from the current atomic-resolution STEM imaging. Several approaches have been used to perform 3D imaging such as electron tomography, which shows excellent results but the shape of the target materials might be limited to nano-particles. The other approach could be optical depth sectioning with a large-illumination angle, where we acquire a series of defocus images along z-direction [1,2]. Recently we have newly installed Delta-type corrector in ARM300CF in the University of Tokyo, and the flat contrast region of Ronchigram is reached to $70 \mathrm{mrad}$ in semi-angle (see Figure 1). It is noteworthy that such high illumination-angle allows us to perform high lateral spatial resolution of $40.5 \mathrm{pm}$ because of the improvement of diffraction limit [3]. Furthermore, it may have a chance to improve depth resolution, maintaining high lateral spatial resolution. With a higher depth resolution electron probe, dynamical diffraction effect could be reduced and therefore electric field imaging [4] with segmented or pixelated detector may be suitable. In this study, we will discuss the technical difficulties and the possibility of large-angle illumination STEM for optical depth sectioning and for the affinity with electric field imaging with our custom made segmented detector.

Figure 1 shows Ronchigrams on amorphous carbon thin films obtained by the respective aberration correctors composed of double and triple dodecapole lenses. With the double dodecapole corrector, an illumination angle is limited to $30 \mathrm{mrad}$, which is limited by six-fold astigmatism. While in a new Deltatype corrector with triple dodecapoles, six-fold astigmatism is well corrected and flat contrast region is extended to $70 \mathrm{mrad}$, as shown in Fig. 1(b). To investigate the depth resolution as a function of illumination angle, we acquired a series of defocus images of nitride and oxide materials, where we investigated the surface atomic structure in a plan-view direction. Figure 2 shows a series of defocus images obtained from $\mathrm{SrTiO}_{3}$ with the illumination angle of $30 \mathrm{mrad}$ at $300 \mathrm{kV}$. At this condition, the depth resolution is not enough to determine the depth location of entrance and exit surfaces. We will discuss in details on the case of large illumination-angle STEM.

In our microscope, a $2^{\text {nd }}$ generation segmented annular all field detector (SAAF, sixteen segment elements) is installed, which enable us to perform electric field imaging at atomic dimensions [5]. Towards quantitative analysis of atomic electric field in materials, it is necessarily to consider dynamical diffraction effect. We also discuss the capability of large-angle illumination for electric field imaging. 


\section{References}

[1] R. Ishikawa et al, Ultramicroscopy 151122 (2015).

[2] R. Ishikawa et al, Appl. Phys. Lett. 109163102 (2016).

[3] S. Morishita et al, Microscopy 6746 (2018).

[4] N. Shibata et al, Nat Phys 8611 (2012).

[5] N. Shibata et al, Nat Commun 715631 (2017).

[6] A part of this work was supported by the Research \& Development Initiative for Scientific Innovation of New Generation Batteries (RISING2).
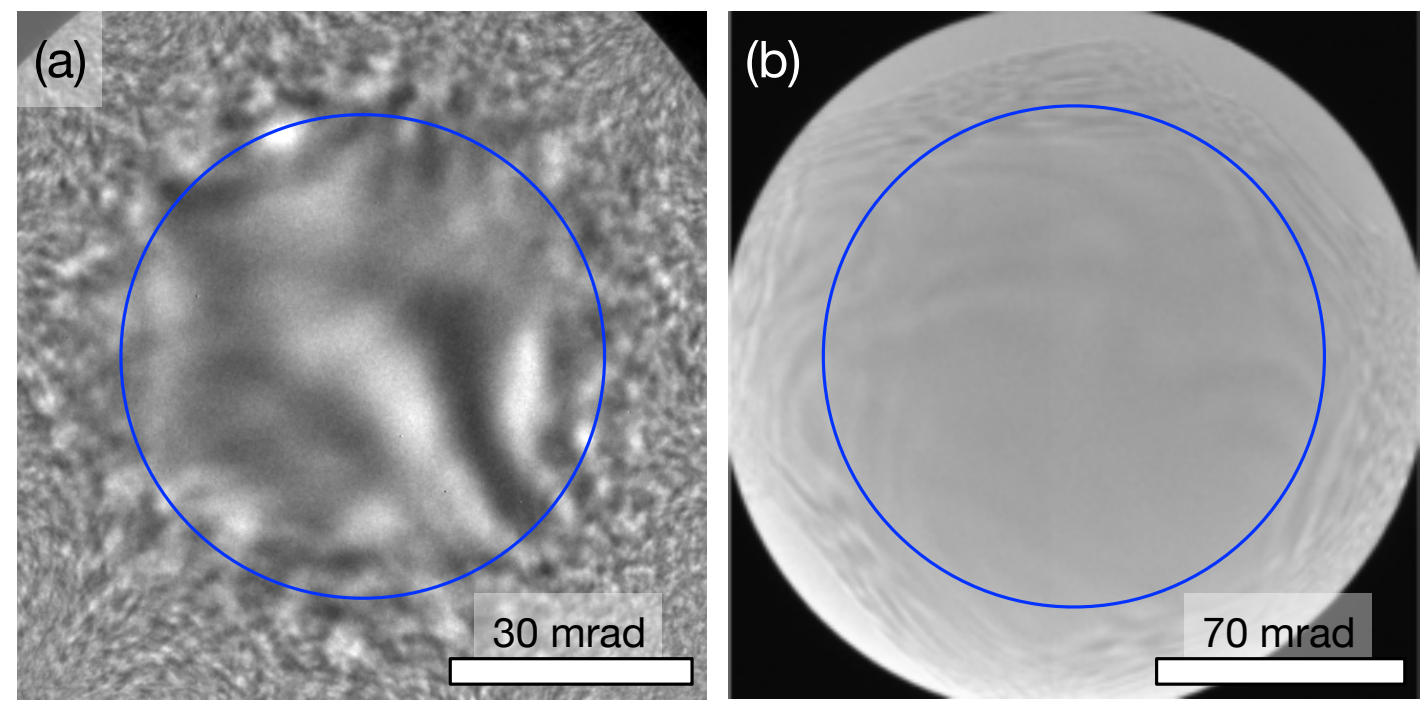

Figure 1. Ronchigrams obtained from amorphous carbon thin films by using (a) double and (b) triple dodecapole aberration corrector systems, respectively.
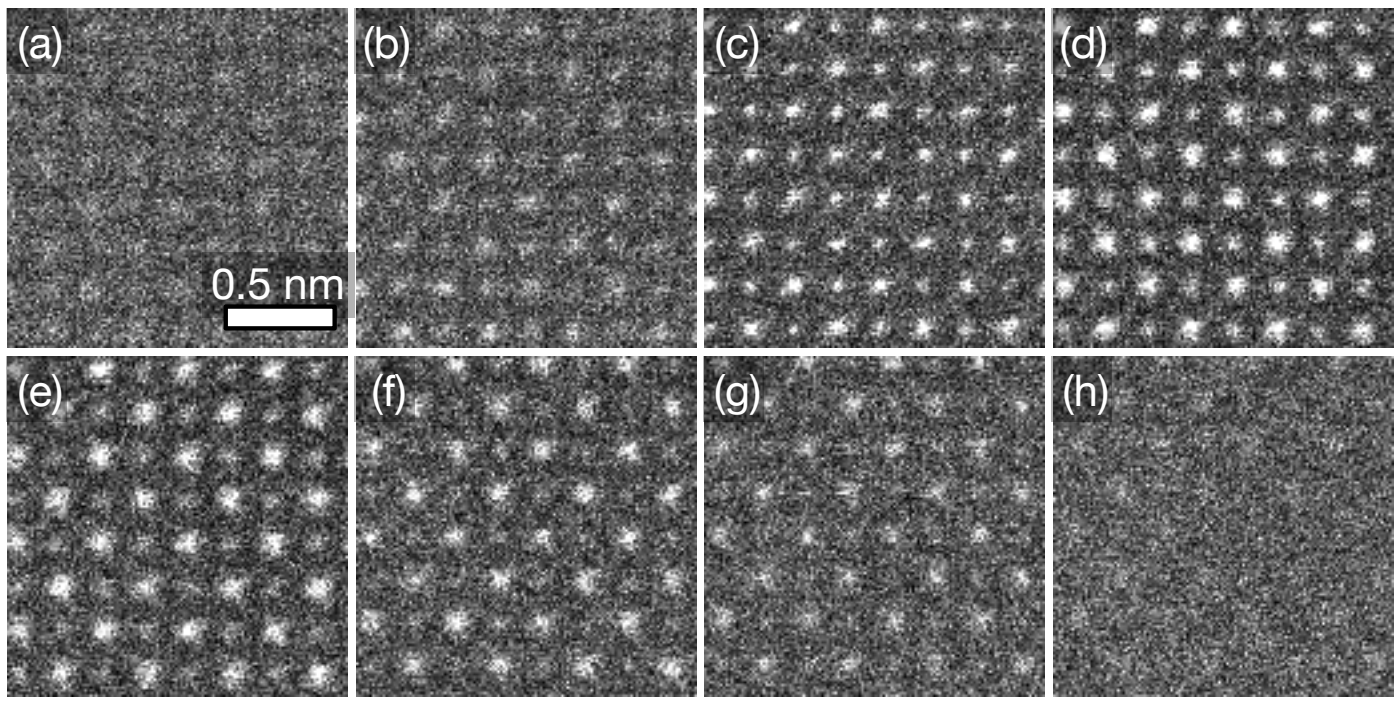

Figure 2. A series of defocus images of $\mathrm{SrTiO}_{3}$ viewed along the [100] direction, where the illumination angle is $30 \mathrm{mrad}$ at $300 \mathrm{kV}$ and the defocus step of these images are $2.9 \mathrm{~nm}$. 